\title{
Ground penetrating radar in crack detection to the forward research
}

\author{
Yi Lin, Zhengping Liu \\ 1.SouthwestJiaotong University, Chengdu 610031 \\ 2.Northwest Research Institute Co.,Ltd of C.R.E.C,LanZhou 730000
}

Keywords: ground penetrating radar, to crack, numerical Simulation.

\begin{abstract}
The ground fissure is hidden hazards of ground deformation.Survey work to crackisthe basicofcontrol measures. Geological radar detection as an emerging technology has advantages of rapid, continuity and visibility; according to a practical example of the detection mechanism of landslide cracks, summed up the ground fissures radar image features. Using numerical simulation method to establish a three-dimensional numerical analysis model, to get to distribution and extension of the crack, the study shows that the results of numerical simulations and field surveys are the same. The results of geological radar detection are accuracy, and provide control of ground fissures a scientific basis.
\end{abstract}

\section{Introduction}

In recent years, the subsidence of the special geological phenomena are frequent in Guangzhou, Shanghai, Shenzhen and other dozens of cities nationwide, triggering a universal concern and heated debate. According to the latest data from China Geological Survey, There are more than 50 cities to have varying degrees of land subsidence and earth fissures in China. Settlement of the area expanded to 94,000 square kilometers, more than 1,400 karst collapse occurred since.

Occurrence of cracks in the local area where exist human activitiesis a geological disaster.It's hidden deeply and lead to some serious problems. We must take effective means to identify the distribution and characteristics of ground fissures, cracks can be completely effective governance.

There aretraditional geophysical methodsincluding seismic imaging,Surface wave and DC probe. Due to the hidden nature of fracture and uncertainty, using traditional physical prospecting method is difficult to identify the spatial distribution. The ground penetrating radar can draw high-precision radar images within a few meters of the surface, with a continuous, rapid, visibility and other features, being more suitable for the detection of concealed ground fissures.

XuXingxin [1] analyzed the application of the principles of geological radar to crack. Through several experiments, ground penetrating radar showed a good detection results for a variety of risks of limestone reservoir. Wang Haiyu, Jiang Tao [2] introduced the technology of detecting cracks and application of results with examples of geological radar to detect cracks. Wu Yuewu et al [3] from the beginning of the vertical cracks mathematical models, from simple to complex, according to the theory of Numerical Simulation - physical simulation - field testing this process on the ground penetrating radar analyzed detecting cracks in the theory, methods and practice effects. Hesummed up eave group characteristics from a single homogeneous medium to homogeneous medium and other various circumstances and the basic method of detecting cracks. Yang Chenglin et al [4] applied ground penetrating radar technology to obtain the high-quality image of reflecting position and depth of the cracks by the example of using ground-penetrating radar detecting Mountains of Sichuan Huaying Zhao Zixiu landslide plasmodium. Studies have shown that the use of ground penetrating radar to detect cracks in the landslide is a convenient and effective method. Early 21's, Wang Yongqiang, Zhang Jichun[5] introduced GPR(Ground Penetrating Radar) to detect mines dump, and From radar detection mechanism and physical characteristics of long cracks excluding demonstrated the applicability and feasibility of the change process. They proposed the use forward modeling of radar to assist image inversion probe. In 2012, Li Yuanqiang[6] by the example of ground penetrating radar to crack introduced workflow of radar, and summarized the GPR waveform characteristics of different genetic types of ground fissures. Through field 
exploration holes, trenches verification, He proved thatthedetection of GPR method is accurate. It provided a scientific basis for analyzing the causes and mechanism of cracks.

\section{Technical Introduction}

GPR technology has become widely accepted in geophysical community as a non-destructive detection technology. Geological radar uses electromagnetic wave reflection principle. By transmitting antenna, it from the ground to the media issued thousands of hertz frequency bands of short pulses of electromagnetic waves. Then after electric differences dielectric interface reflection effect,the reflected wave is received by ground antennas. Thenradar post-processing software begins signal processing. Data processing includes removing bad sectors, zero calibration, filtering, offset and etc. Finally,obtaining subsurface radar observed a longitudinal section view,the abscissa is the ground position, and the ordinate is the pulse duration. According to receive the reflected wave trip time, intensity, waveform and antenna position, we can determine the characteristics of subsurface and underground structures.

There are significantly different electromagnetic parameters between bedrock and air in the cracks. Electromagnetic wave propagation velocity depends on the dielectric constant of the medium. Electromagnetic wave velocity $v(\mathrm{~m} / \mathrm{ns})$ can be expressed as:

$$
v=\frac{c}{\sqrt{\varepsilon}}
$$

In the above formula, $\varepsilon(\mathrm{F} / \mathrm{m})$ for the relative dielectric constant of the medium; $\mathrm{c}=3 \times 108 \mathrm{~m} /$ $\mathrm{s}$ for the vacuum of electromagnetic wave propagation velocity.

Reflection of high-frequency electromagnetic waves occurs in the difference between the two electric medium. This difference in power is measured by the relative permittivity. The strength of the reflected waves to measure the reflection coefficient R:

$$
R=\frac{\sqrt{\varepsilon_{2}}-\sqrt{\varepsilon_{1}}}{\sqrt{\varepsilon_{2}}+\sqrt{\varepsilon_{1}}} .
$$

(2) $\varepsilon 1$ and $\varepsilon 2$ are two different media relative permittivity. The greater the difference in relative dielectric constant of the medium, the reflection is more intense. Ground penetrating radar detect the target body on the surface by using electrical differences.

Radar detection depth depends on the electromagnetic wave penetration. When the electromagnetic wave propagate in a loss medium, penetration depends on the attenuation coefficient $\alpha$. The larger the attenuation coefficient is,the more energy loss. The depth is Shallower. The attenuation coefficient $\alpha$ can be expressed as:

$$
\alpha=Z_{0} \frac{\sigma}{2 \sqrt{\varepsilon}}
$$

$Z 0=377 \Omega$ impedance of free

(3)

space; $\sigma(\mathrm{ms} / \mathrm{m})$ for medium conductivity. You can see the impact of the attenuation coefficient by the medium conductivity and relative dielectric constant. Additionally, ground wave penetration is also associated with the frequency of the antenna. Due to the different types of cracks, When probe according to local conditions and terrain characteristics of ground fissures, were the instrument selection, antenna configuration and parameter settings.

The chaos Shek Kong in Hanyuan detection used IDS Italian company RIS-K2 portable ground penetrating radar. $40 \mathrm{MHz}$ unshielded antenna configuration, spacing $3 \mathrm{~m}$, measuring $0.25 \mathrm{~m}$ pitch,measuring use profiling method.It is transmit and receive antennas at a constant pitch along a survey line synchronous mobile measurement. 


\section{Numerical Simulation}

In order to verify the usefulness of radar to detect cracks,modeling based on field testing,modeling and forward profile is shown in Figure 1 . Model size is $200 \mathrm{~m} \times 40 \mathrm{~m}$, and crack width is $10 \mathrm{~cm} .4 \mathrm{~m}-12 \mathrm{~m}$ below ground level. Permittivity and conductivity as shown in figure:
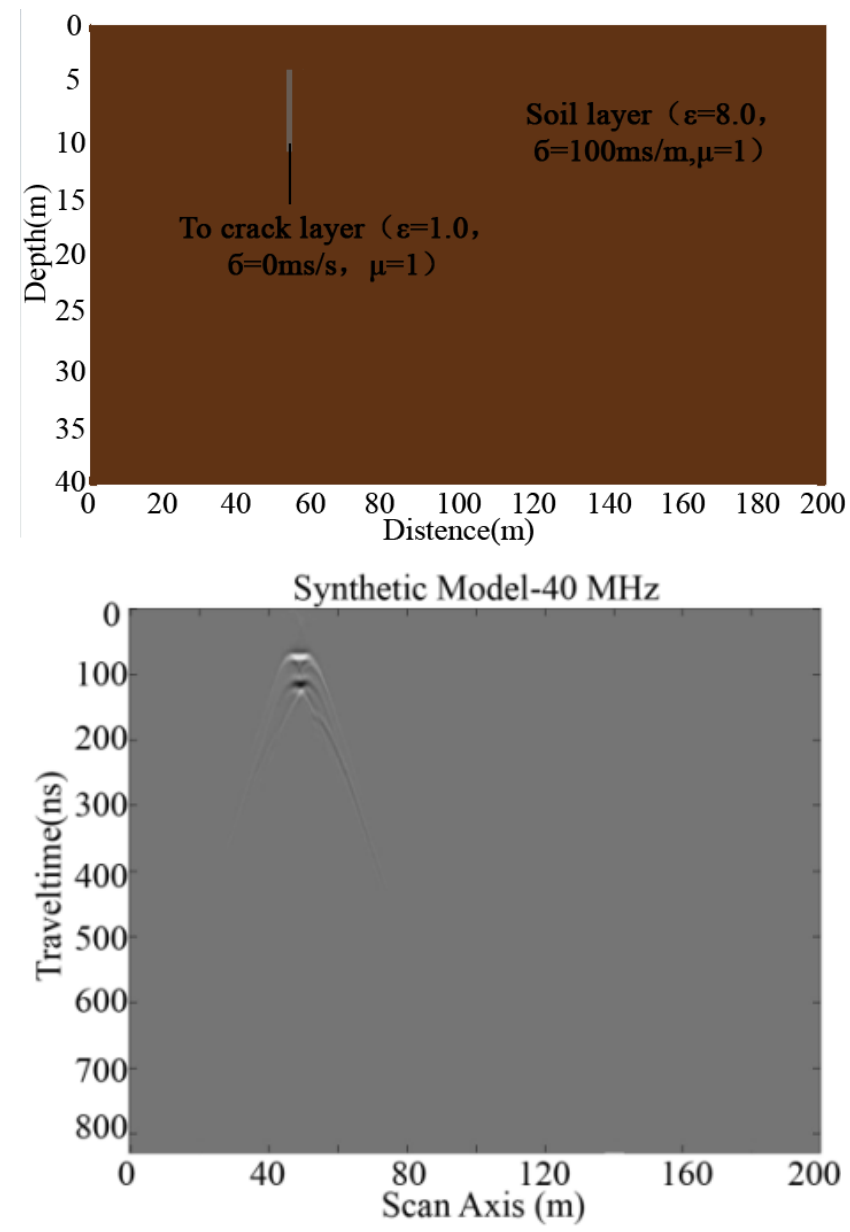

Fig. 1 Established model and forward profile

Established the thickness of layers, the physical parameters and the width of ground fissure are refer to the actual model. The results of forward are very close to the actual profile.It showsthat the direct wave of soil at the top of the fissure changes, occurringhoroscopes diffraction. Forward results showed radar probing ground fissure is accurate and reliable.

\section{Projects}

Stony post on the territory of the city of Ya'an City Hanyuan,and located in the northern section of the eastern edge of the mountain range. Terrain is four-week high middle low. Four weeks surrounded by mountains. Terraingives priority toMountains, low mountains, hillsandterraces.Slope is relatively moderate. Usually, it is $15^{\circ}$ to $35^{\circ}$.In its territory, there are a number of rivers, abundant water resources and lush vegetation.Lithology mainly is fine sandstone, shale, sandstone and seams. Rich in mineral resources surrounding,there are granite limestone clay mineral ore and coal, etc. Due to frequent mining, it formed gob areaof different sizes anduncertain position. Geological structure in Chaos Shek Kong landslide area is complex.There is gob area in front of landslide. Rock ruptured, and ground fissures are widely distributed.

Figure 2 shows the GPR measurements in the chaos Shek Kong landslide area.The survey line is measured from the cement road edge.Crossing concrete road, it is extended to the roadside grass to arrange, and it is flat. Through a crack in the ground at $60 \mathrm{~m}$,from the radar image analysis, shallow phase axis $0 \sim 10 \mathrm{~m}$ clear break.It showed that the premises are close to vertical fracture zone. After 
exploratory verification, there is gob area in the depth of 35m. Upper rock stresschanges on the gob area.Finally it leads to cracks.

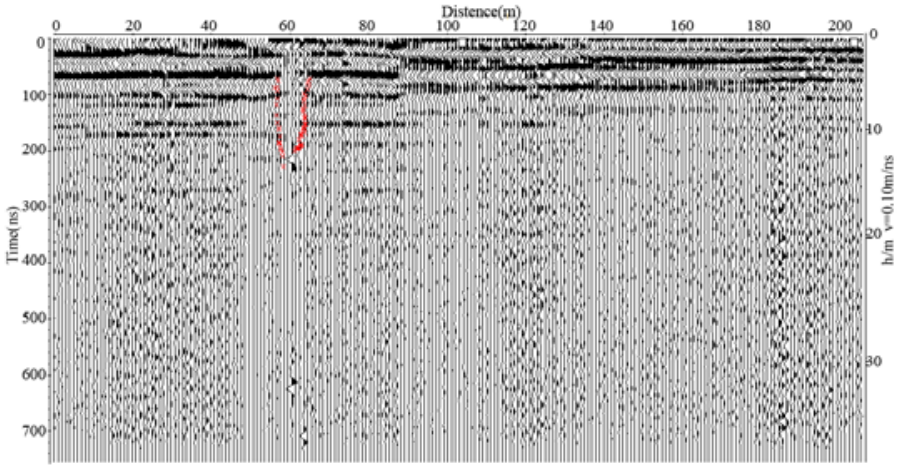

Fig. 2Radar cross sectional view of the ground fissures in the chaos Shek Kong

\section{Conclusion}

Using ground-penetrating radar to detect ground fissures, understanding the principle of detection is first. Through on-site survey, arrange rational survey lines, and set the parameters and select parameters of subsurface physical properties. Only dothatcan we obtain high-quality radar profilesand accurate explanation. Proved by numerical simulation, geological radar has the ability to distinguish, high observing efficiency, continuity and other advantages. Used to detect ground fissure is feasible and effective.

\section{References}

[1]XuXingxin, WuJing, WuXiangan, LifuCai, et al, A Research on GPR-Detection of Reservoirs Hidden-dangers and leakage channel in Limestone Region, J. Resources and Hydropower Engineering. 30 (1999) 45-47

[2] Wang Yuhai, Jiang Tao, Application of georadar in the examination of ground cracks, J. Investigation Science and Technology. 4 (2001) 62-64

[3]FanYuewu, LiXiuzhong, BaiXingying , ChengMingdao, Study on highway structure layer crack detection by ground penetrating radar, J. Highway and Transportation Research. 31 (2007)1-7.

[4] YangChenglin, ChenNingsheng, ShiLeilei,Application of ground penetrating radar in the explorationof landslide fissures inmtzhaozixiu, J. Geophysical and Geochemical Exploration. 32 (2008) 221-224.

[5]Wang Yongqiang and Zhang Jichun:Mine dump crack detection method based on radar technology (2010)

[6]Li Yuanqiang, a few examples of Ground penetrating radar to crack, J. 36(2012) 652-654 